\title{
VANISHING SPECIFIC HEAT \\ FOR THE CLASSICAL SOLUTIONS \\ OF A MULTIDIMENSIONAL STEFAN PROBLEM WITH KINETIC CONDITION
}

\author{
$\mathrm{BY}$ \\ FAHUAI YI (Department of Mathematics, Suzhou University, Suzhou, China) \\ AND \\ JINDUO LIU (North China Mining College, Yanjiao, Beijing, China)
}

\begin{abstract}
In this paper we prove that the multidimensional Hele-Shaw problem with kinetic condition at the free boundary is the limit case of the Stefan problem with kinetic condition at the free boundary in the classical sense when the specific heat $\varepsilon$ goes to zero. The method is the use of a fixed point theorem; the key step is to construct a suitable function space in which we can get the existence and uniform estimates with respect to $\varepsilon>0$ at the same time as for classical solutions of the multidimensional Stefan problem with kinetic condition at the free boundary. For the sake of simplicity, we only consider one-phase problems in three space dimensions, although the method used here is also applicable for two-phase problems and any space dimensions.
\end{abstract}

1. Introduction. Suppose $\Omega \subset \mathbb{R}^{3}$ is a bounded annual domain with $\partial \Omega=\Gamma_{0} \cup \Gamma_{1}$; here $\Gamma_{0}$ is the outside boundary of $\Omega$ and $\Gamma_{1}$ is the inside one. Let $\bigcup_{0 \leq t \leq T} \Gamma(t)$ be the (unknown) free boundary with $\Gamma(0)=\Gamma_{0}$ and $\Gamma_{1 T}=\Gamma_{1} \times[0, T]$. Denote the domain between $\Gamma(t)$ and $\Gamma_{1}$ by $\Omega(t)$.

The Stefan problem with kinetic condition at the free boundary is to find a temperature field $u(x, t), x \in \Omega(t), 0<t \leq T$, and a free boundary $\bigcup_{0<t \leq T} \Gamma(t)$, satisfying (see [1])

$$
\begin{aligned}
\varepsilon \partial_{t} u-\Delta u=0, & x \in \Omega(t), 0<t \leq T, \\
u=g(x, t) & \text { on } \Gamma_{1 T}, \\
u=V_{n} & \text { on } \Gamma(t), 0 \leq t \leq T, \\
V_{n}=-\frac{\partial u}{\partial n} & \text { on } \Gamma(t), 0 \leq t \leq T, \\
u(x, 0)=u_{0}(x) & \text { on } t=0,
\end{aligned}
$$

Received November 4, 1997.

1991 Mathematics Subject Classification. Primary 35R35.

Key words and phrases. Vanishing specific heat, Stefan problem, Kinetic condition.

(C)1999 Brown University 
where $\varepsilon>0$ is the specific heat, $n$ is the unit normal on $\Gamma(t)$ towards the outside of $\Omega(t)$ and $V_{n}$ is the normal velocity in the $n$ direction. $g(x, t)$ and $u_{0}(x)$ are known functions.

For fixed $\varepsilon>0$, the problem (1.1) is called the Stefan problem with kinetic condition. At the one-space-dimensional case this problem had been considered in [1] and [2], and, recently, with special two-dimensional geometries in [3] or, under strong technical conditions in [4] for any space dimensions by using Nash's implicit function theorem as in $[5]$.

For $\varepsilon=0$, the problem (1.1) without initial condition is called the Hele-Shaw problem. Its classical existence in the two-dimensional case was considered in [6] and [7], respectively, in Hölder space and in analytic function space.

In this work we prove that for fixed $\varepsilon>0$ the problem has a unique classical solution. At the same time we get uniform estimates with respect to $\varepsilon>0$ which ensure that the solutions converge to the solution with $\varepsilon=0$ when $\varepsilon$ goes to zero. So our result also provides a proof of the classical existence of a local solution for the Hele-Shaw problem with kinetic condition at the free boundary in any space dimensions.

The final session of the European Scientific Foundation meeting on "Problems with Regularized Free Boundaries" (Oxford, December 1993) was a general discussion of ten open questions related to the Hele-Shaw problem that the participants thought particularly interesting. Our problem arises from the ninth one (see [8]) which asks if the zero-specific-heat limit in the classical sense of the Stefan problem is the Hele-Shaw problem. In [9] we had proved that the Hele-Shaw problem with the Gibbs-Thomson relation which includes surface tension as well as a kinetic condition at the free boundary is the zero-specific-heat limit in the classical sense of the Stefan problem with the same free boundary conditions in any space dimensions.

Following the idea of [5], we introduce a (unknown) distance function $\rho(\omega, t), \omega=$ $\left(\omega_{1}, \omega_{2}\right) \in \mathbb{R}^{2}, t>0$, to describe the free boundary. More precisely, suppose $\Gamma_{1} \cap \Gamma_{0}=\varnothing$. For the points on the surface $\Gamma_{0}$, we introduce coordinates $\omega=\left(\omega_{1}, \omega_{2}\right)$; we also denote by $x(\omega) \in \Gamma_{0}$ and $n(\omega)$ the unit exterior normal to $\Gamma_{0}$.

Let $\gamma_{0}$ be a given positive number such that the surface $\left\{x=x(\omega) \pm n(\omega) \gamma, 0<\gamma<\gamma_{0}\right\}$ has no self-intersection and does not intersect $\Gamma_{1}$. Let $\rho(\omega, t)$ be a smooth function defined on $\Gamma_{0} \times[0, T]$ such that $\rho(\omega, 0)=0$ and $\max |\rho(\omega, t)| \leq \gamma_{0} / 4$. We denote by $\Omega_{\rho T}$ the region bounded by the planes $t=0, T$, surface $\Gamma_{1 T}$ and $\Gamma_{\rho T}=\{(x, t): x=x(\omega)+\rho(\omega, t) n(\omega)\}$. The problem (1.1) can be written as follows:

$$
\begin{array}{ll}
\varepsilon \partial_{t} u-\Delta u=0 & \text { in } \Omega_{\rho T}, \\
u=g(x, t) & \text { on } \Gamma_{1 T}, \\
\frac{\partial u}{\partial n}+u=0 & \text { on } \Gamma_{\rho T}, \\
V_{n}=u & \text { on } \Gamma_{\rho T}, \\
u(x, 0)=u_{0}(x) & \text { on } t=0 .
\end{array}
$$

In the next section we use a Hanzawa diffeomorphism to change the problem (1.2)(1.6) into a cylindrical domain and straighten the free boundary. In Sec. 3, we prove the existence of a solution to problem (1.2)-(1.6) and get a uniform estimate with respect 
to $\varepsilon>0$ following the ideas in [6] and [9]. In the last section, we prove the convergence theorem.

2. Straighten the free boundary and main results. To prove the solvability of the problem (1.2)-(1.6), it is convenient to reduce it to a problem in a fixed domain. To this end, we use the Hanzawa diffeomorphism presented in [5]. Suppose $\gamma_{0}$ introduced above is so small that the mapping $x: \Gamma_{0} \times\left[-\gamma_{0}, \gamma_{0}\right] \rightarrow \mathbb{R}^{3}$ defined by the rule $x(\omega, \lambda)=$ $x(\omega)+\lambda n(\omega)$ is regular and one-to-one. Let the range of this mapping be

$$
N_{0}=\left\{x(\omega, \lambda) ;(\omega, \lambda) \in \Gamma_{0} \times\left[-\gamma_{0}, \gamma_{0}\right]\right\} .
$$

The inverse mapping from $N_{0}$ to $\Gamma_{0} \times\left[-\gamma_{0}, \gamma_{0}\right]$ is defined as follows: $x \rightarrow(\omega(x), \lambda(x))$. We set

$$
\begin{aligned}
& \varphi^{(i)}(\omega, \lambda)=\left.\nabla_{x} \omega_{i}(x)\right|_{x=x(\omega, \lambda)}, i=1,2, \\
& \varphi^{(3)}(\omega, \lambda)=\left.\nabla_{x} \lambda(x)\right|_{x=x(\omega, \lambda)},
\end{aligned}
$$

from which we can see that $\partial \Omega \in C^{3+\alpha}$ reduces to $\varphi^{(i)}(\omega, \lambda) \in C^{2+\alpha}\left(\Gamma_{0} \times\left[-\gamma_{0}, \gamma_{0}\right]\right)$, $i=1,2,3$. We shall show below that for sufficiently small $T$, the free boundary surface $\Gamma_{\rho T}$ can be described by the equation

$$
h_{\rho}(x, t) \equiv \lambda(x)-\rho(\omega(x), t)=0 .
$$

This makes it possible to compute the normal velocity for $\Gamma(t)$ :

$$
V_{n}(\omega, t)=\left.\frac{\partial_{t} \rho}{\left|\nabla_{x} h_{\rho}\right|}\right|_{x=x(\omega, \rho(\omega, t))} .
$$

We note that by construction $\varphi^{(3)}(\omega, 0)=n(\omega)$ is orthogonal to $\varphi^{(j)}(\omega, 0), j=1,2$.

Further, let $\chi(\lambda) \in C^{\infty}\left(\left[-\gamma_{0}, \gamma_{0}\right]\right)$ be such that

$$
\begin{aligned}
\chi(\lambda) & =0, \text { in }|\lambda|>\frac{3}{4} \gamma_{0}, \\
\chi(\lambda) & =1, \text { in }|\lambda|<\frac{1}{4} \gamma_{0}, \\
\chi^{\prime}(\lambda) & \leq 3 \gamma_{0}^{-1} .
\end{aligned}
$$

Then $1+\chi^{\prime}(\lambda) \mu \geq \frac{1}{4}$ if $|\mu| \leq \gamma_{0} / 4$.

For any $\rho(\omega, t) \in C^{2,1}\left(\Gamma_{0 T}\right)$, with $\left.\rho\right|_{t=0}=0$ and $\max _{\Gamma_{0 T}}|\rho|<\gamma_{0} / 4$, we define the Hanzawa diffeomorphism

$$
\ell_{\rho T}: \mathbb{R}_{y}^{3} \times[0, T] \rightarrow \mathbb{R}_{x}^{3} \times[0, T]
$$

in the manner $(x, t)=\ell_{\rho T}(y, t)$ with

$$
\begin{cases}x=y, & \text { if } \operatorname{dist}\left(y, \Gamma_{0}\right) \geq \frac{3}{4} \gamma_{0}, \\ x=x(\omega)+[\eta+\chi(\eta) \rho(\omega, t)] n(\omega), & \text { if } \operatorname{dist}\left(y, \Gamma_{0}\right) \leq \frac{3}{4} \gamma_{0}\end{cases}
$$

where $y=y(\omega, \eta)$ is in the neighborhood $N_{0}$ of $\Gamma_{0}$ and $(\omega, \eta)$ are local coordinates of $y$ in $N_{0}$. In local coordinates of $N_{0} \times[0, T]$, we have

$$
\ell_{\rho T}(\omega, \eta ; t)=(\omega, \eta+\chi(\eta) \rho(\omega, t) ; t) \equiv(\omega, \lambda ; t),
$$

where $\lambda=\eta+\chi(\eta) \rho(\omega, t)$. 
The transformation $\ell_{\rho T}^{-1}$ takes the noncylindrical domain $\Omega_{\rho T}$ to the cylindrical domain $\Omega_{T}=\Omega \times[0, T]$. In the following, we make the change of variables $(x, t)=\ell_{\rho T}(y, t)$ and let

$$
v(y, t)=u\left(\ell_{\rho T}(y, t)\right)
$$

Notice that

$$
\frac{\partial u}{\partial n}=\left(\left|\nabla_{x} h_{\rho}\right|^{2} \partial_{\eta} v-\sum_{i=1}^{2} \partial_{\omega_{i}} \rho\left|\varphi^{(i)}(\omega, \rho)\right|^{2} \partial_{\omega_{i}} v\right) /\left|\nabla_{x} h_{\rho}\right|
$$

If we define

$$
S_{\rho} \equiv\left|\nabla_{x} h_{\rho}\right|^{2}=1+\left|\sum_{i=1}^{2} \partial_{\omega_{i}} \rho \varphi^{(i)}(\omega, \rho)\right|^{2},
$$

then problem (1.2)-(1.6) becomes

$$
\begin{array}{ll}
\varepsilon \partial_{t} v-\mathcal{L}_{\rho} v=0 & \text { in } \Omega_{T}, \\
v=g(y, t) & \text { on } \Gamma_{1 T}, \\
v(y, 0)=u_{0}(y) & \text { on } t=0, \\
S_{\rho} \partial_{\eta} v-\sum_{i=1}^{2} \partial_{\omega_{i}} \rho\left|\varphi^{(i)}(\omega, \rho)\right|^{2} \partial_{\omega_{i}} v+S_{\rho}^{1 / 2} v=0 & \text { on } \Gamma_{0 T}, \\
\partial_{t} \rho=S_{\rho}^{1 / 2} v & \text { on } \Gamma_{0 T} .
\end{array}
$$

Here

$$
\begin{aligned}
\mathcal{L}_{\rho} & =\sum_{i, j=1}^{3} a_{\rho}^{i j} \frac{\partial^{2}}{\partial y_{i} \partial y_{j}}+\sum_{i, j=1}^{3} a_{\rho}^{i} \frac{\partial}{\partial y_{i}}, \\
a_{\rho}^{i j} & =\left.\nabla_{x}\left(\ell_{\rho T}^{-1}\right)_{i} \cdot \nabla_{x}\left(\ell_{\rho T}^{-1}\right)_{j}\right|_{(x, t)=\ell_{\rho T}(y, t)} \\
& =a_{i j}\left(\rho, \nabla_{\omega} \rho\right) \\
a_{\rho}^{i} & =\left[\varepsilon \partial_{t}\left(\ell_{\rho T}^{-1}\right)_{i}-\left.\Delta_{x}\left(\ell_{\rho T}^{-1}\right)_{i}\right|_{(x, t)=\ell_{\rho T}(y, t)}\right. \\
& =a_{i}\left(\rho, \nabla_{\omega} \rho, D_{\omega}^{2} \rho, \varepsilon \partial_{t} \rho\right),
\end{aligned}
$$

and $\left.a_{\rho}^{i j}\right|_{t=0}=\delta_{i j}, \delta_{i j}=1$ if $i=j, \delta_{i j}=0$ for $i \neq j,\left.a_{\rho}^{i}\right|_{t=0}=0$ because the transformation $\ell_{\rho T}$ is the identity at $t=0$.

For $0<\alpha<1$, define the function space of $\rho(\omega, t)$,

$$
\begin{aligned}
D=\{\rho \in & \left.C^{2+\alpha, 1+\alpha / 2}\left(\Gamma_{0 T}\right)|\rho(\omega, 0)=0,| \rho\right|_{L^{\infty}\left(\Gamma_{0 T}\right)} \leq \gamma_{0} / 4, \\
& |\rho|_{B\left([0, T] ; C^{2+\alpha}\left(\Gamma_{0}\right)\right.} \leq M_{0},\left|\partial_{t} \rho\right|_{L^{\infty}\left(\Gamma_{0 T}\right)} \leq 2 M_{0}, \\
& \left.\left|D_{\omega}^{2} \rho\right|_{C^{\alpha, \alpha / 2}\left(\Gamma_{0 T}\right)} \leq N,\left|\varepsilon^{1+\alpha / 2} \partial_{t} \rho\right|_{C^{\alpha, \alpha / 2}\left(\Gamma_{0 T}\right)} \leq N\right\},
\end{aligned}
$$

where $\gamma_{0}$ is determined before, $B\left([0, T] ; C^{2+\alpha}\left(\Gamma_{0}\right)\right)$ denotes the Banach space of bounded functions from $[0, T]$ with values in $C^{2+\alpha}\left(\Gamma_{0}\right), M_{0}=|g|_{L^{\infty}\left(\Gamma_{0 T}\right)}+\left|u_{0}\right|_{L^{\infty}(\Omega)}$, and $T$ and $N$ are to be determined later on. We will find that the choice of $N$ depends only on known data and is independent of the choice of $T$. Set

$$
|\rho|_{D}=|\rho|_{B\left([0, T] ; C^{2+\alpha}\left(\Gamma_{0}\right)\right.}+\left|\partial_{t} \rho\right|_{L^{\infty}\left(\Gamma_{0 T}\right)}+\left|D_{\omega}^{2} \rho\right|_{C^{\alpha, \alpha / 2}\left(\Gamma_{0 T}\right)}+\left|\varepsilon^{1+\alpha / 2} \partial_{t} \rho\right|_{C^{\alpha, \alpha / 2}\left(\Gamma_{0 T}\right)} .
$$


It is seen that $D$ is a closed convex set in the Banach space $C^{2+\alpha, 1+\alpha / 2}\left(\Gamma_{0 T}\right)$. Notice that for any $\xi \in \mathbb{R}^{3}$,

$$
\sigma_{1}|\xi|^{2} \leq a_{\rho}^{i j} \xi_{i} \xi_{j} \leq \sigma_{2}|\xi|^{2}
$$

where the positive numbers $\sigma_{1}$ and $\sigma_{2}$ do not depend on $\varepsilon$. Suppose

$$
\begin{aligned}
& \Gamma_{0} \in C^{3+\alpha}, \\
& g(x, t) \in C^{2+\alpha, 1+\alpha / 2}\left(\Gamma_{1 T}\right), \\
& u_{0}(x) \in C^{2+\alpha}(\bar{\Omega}),
\end{aligned}
$$

and the consistency conditions

$$
\begin{array}{ll}
\partial_{n} u_{0}+u_{0}=0 & \text { on } \Gamma_{0}, \\
g(x, 0)=u_{0}(x) & \text { on } \Gamma_{1}, \\
\partial_{t} g(x, 0)=\Delta u_{0}(x)=0 & \text { on } \Gamma_{1} .
\end{array}
$$

Theorem 2.1. Under the conditions (2.6)-(2.11), for fixed $\varepsilon>0$, the problem (2.1)(2.5) has a unique solution $\rho=\rho_{\varepsilon} \in D$.

Here we suppose that $\rho$ is the unique unknown because once $\rho$ is known, then $v$ can be determined.

3. The proof of Theorem 2.1. The idea of the proof for the uniqueness is the same as in [6]; we omit the details.

For given $\rho \in D, \ell_{\rho T}$ is well-defined. Let $v(y, t)$ be the solution of $(2.1)-(2.4)$ that depends on $\rho$ and $\varepsilon$. It is obvious that for fixed $\varepsilon>0$ and given $\rho$, problem (2.1)-(2.4) has a unique solution $v(y, t) \in C^{2+\alpha, 1+\alpha / 2}\left(\bar{\Omega}_{T}\right)$. In order to get a uniform estimate with respect to $\varepsilon>0$ we let, for $0 \leq t \leq T$,

$$
t=\varepsilon \tau, \quad 0 \leq \tau \leq \varepsilon^{-1} T \equiv T_{\varepsilon},
$$

define $h(\omega, \tau)=\rho(\omega, \varepsilon \tau)$ and $W(y, \tau)=v(y, \varepsilon \tau)$; therefore $\partial_{\tau} h=\varepsilon \partial_{t} \rho$ and $\partial_{\tau} W=\varepsilon \partial_{t} v$.

Under the change (3.1), problem (2.1) $-(2.4)$ becomes

$$
\begin{array}{ll}
\partial_{\tau} W-\mathcal{L}_{h} W=0 & \text { in } \Omega_{T_{\varepsilon}} \\
W(y, \tau)=g(y, \varepsilon \tau) \equiv G(y, \tau) & \text { on } \Gamma_{1 T_{\varepsilon}} \\
W(y, 0)=u_{0}(y) & \text { on } t=0 \\
S_{h} \partial_{\eta} W-\sum_{i=1}^{2} \partial_{\omega_{i}} h\left|\varphi^{(i)}(\omega, h)\right|^{2} \partial_{\omega_{i}} W+S_{h}^{1 / 2} W=0 & \text { on } \Gamma_{0 T_{\varepsilon}}
\end{array}
$$

where

$$
\begin{aligned}
\mathcal{L}_{h} & =\sum_{i, j=1}^{3} a_{h}^{i j} \frac{\partial^{2}}{\partial y_{i} \partial y_{j}}+\sum_{i=1}^{3} a_{h}^{i} \frac{\partial}{\partial y_{i}} \\
a_{h}^{i j} & =a_{i j}\left(h, \nabla_{\omega} h\right), \\
a_{h}^{i} & =a_{i}\left(h, \nabla_{\omega} h, D_{\omega}^{2} h, \partial_{\tau} h\right) .
\end{aligned}
$$


Considering

$$
\frac{\left|\partial_{\tau} h\left(\omega, \tau_{1}\right)-\partial_{\tau} h\left(\omega, \tau_{2}\right)\right|}{\left|\tau_{1}-\tau_{2}\right|^{\alpha / 2}}=\frac{\varepsilon\left|\partial_{t} \rho\left(\omega, t_{1}\right)-\partial_{t} \rho\left(\omega, t_{2}\right)\right|}{\varepsilon^{-\alpha / 2}\left|t_{1}-t_{2}\right|^{\alpha / 2}},
$$

we have $\partial_{\tau} h \in C^{\alpha, \alpha / 2}\left(\Gamma_{0 T_{\varepsilon}}\right)$, and by the definition of the function set $D$ we know

$$
\begin{aligned}
& \left|h, \nabla_{\omega} h, D_{\omega}^{2} h, \partial_{\tau} h\right|_{L^{\infty}\left(\Omega_{T_{\varepsilon}}\right)} \leq 3 M_{0}, \\
& \left|h, \nabla_{\omega} h, D_{\omega}^{2} h, \partial_{\tau} h\right|_{C^{\alpha, \alpha / 2}\left(\bar{\Omega}_{T_{\varepsilon}}\right)} \leq 3\left(M_{0}+N\right) ;
\end{aligned}
$$

therefore,

$$
\begin{aligned}
& |W|_{L^{\infty}\left(\Omega_{T_{\varepsilon}}\right)} \leq M_{0} \quad \text { (by the maximum principle) } \\
& |W|_{C^{\alpha, \alpha / 2}\left(\bar{\Omega}_{T_{\varepsilon}}\right)} \leq C\left(M_{0}\right) M_{\alpha}=C\left(M_{\alpha}\right) \\
& |W|_{C^{2+\alpha, 1+\alpha / 2}\left(\bar{\Omega}_{T_{\varepsilon}}\right)} \leq C\left(M_{0}, N\right) M_{2+\alpha}=C\left(M_{2+\alpha}, N\right)
\end{aligned}
$$

where

$$
\begin{aligned}
M_{0} & =|G|_{L^{\infty}\left(\Gamma_{1 T_{\varepsilon}}\right)}+\left|u_{0}\right|_{L^{\infty}(\Omega)} \\
& =|g|_{L^{\infty}\left(\Gamma_{1 T}\right)}+\left|u_{0}\right|_{L^{\infty}(\Omega)}, \\
M_{\alpha} & =|G|_{C^{\alpha, \alpha / 2}\left(\Gamma_{1 T \varepsilon}\right)}+\left|u_{0}\right|_{C^{\alpha}(\bar{\Omega})} \\
& \leq|g|_{C^{\alpha, \alpha / 2}\left(\Gamma_{1 T}\right)}+\left|u_{0}\right|_{C^{\alpha}(\bar{\Omega})}, \\
M_{2+\alpha} & =|G|_{C^{2+\alpha, 1+\alpha / 2}\left(\Gamma_{1 T_{\varepsilon}}\right)}+\left|u_{0}\right|_{C^{2+\alpha}(\bar{\Omega})} \\
& \leq|g|_{C^{2+\alpha, 1+\alpha / 2}\left(\Gamma_{1 T}\right)}+\left|u_{0}\right|_{C^{2+\alpha}(\bar{\Omega})}
\end{aligned}
$$

if $\varepsilon \leq 1$. $C\left(M_{\alpha}\right)$ and $C\left(M_{2+\alpha}, N\right)$ do not depend on $T_{\varepsilon}$ because the maximum of $|W|$ does not depend on $T_{\varepsilon}$, and other seminorms in $C^{2+\alpha, 1+\alpha / 2}\left(\bar{\Omega}_{T_{\varepsilon}}\right)$ depend on the maximum of $|W|$ but they are independent of $T_{\varepsilon}$ according to the parabolic theorem. The estimates (3.6)-(3.8) indicate that

$$
\begin{aligned}
& |v|_{L^{\infty}\left(\Omega_{T}\right)} \leq M_{0}, \\
& |v|_{B\left([0, T] ; C^{\alpha}(\bar{\Omega})\right)}+\left|\varepsilon^{\alpha / 2} v\right|_{C^{\alpha, \alpha / 2}\left(\bar{\Omega}_{T}\right)} \leq C\left(M_{\alpha}\right), \\
& |v|_{B\left([0, T] ; C^{2+\alpha}(\bar{\Omega})\right)} \leq C\left(M_{2+\alpha}, N\right) .
\end{aligned}
$$

The next step is to find a $\bar{\rho}(\omega, t)$ by the condition (2.5). For known $\rho \in D$ and $v$ determined above, let $\bar{\rho}$ be the solution of the periodic Cauchy problem of the first-order partial differential equation

$$
\begin{gathered}
\partial_{t} \bar{\rho}=v(\omega, t) \sqrt{1+\sum_{i=1}^{2}\left|\varphi^{(i)}(\omega, \rho)\right|^{2} \bar{\rho}_{\omega_{i}}^{2}} \text { in } \Gamma_{0 T} \\
\bar{\rho}(\omega, 0)=0 \quad \text { on } t=0 .
\end{gathered}
$$

Here we used the relation $\varphi^{(1)} \cdot \varphi^{(2)}=0$ (see [10]) and denote $\left.v(\omega, t) \equiv v(x, t)\right|_{x \in \Gamma_{0}}$ by $v(\omega, t)$.

Lemma 3.1. There exists a $T>0$ such that for each $\rho \in D$, there exists a unique solution $\bar{\rho} \in D$ to the problem (3.12) and (3.13). 
The proof of Lemma 3.1. The uniqueness can easily be seen by observing that the difference of two solutions of (3.12) and (3.13) satisfies a linear first-order equation.

Let $\varphi_{\delta}^{(i)}, i=1,2$ and $v_{\delta}$ be the molifications of $\varphi^{(i)}(\omega, \rho(\omega, t))$ and $v(\omega, t)$ in the variable $\omega=\left(\omega_{1}, \omega_{2}\right)$, so that

$$
\begin{aligned}
& \left|\varphi_{\delta}^{(i)}-\varphi^{(i)}\right|_{D} \rightarrow 0 \quad(\text { as } \delta \rightarrow 0), \quad i=1,2, \\
& \left|v_{\delta}-v\right|_{B\left([0, T] ; C^{2+\alpha}\left(\Gamma_{0}\right)\right)} \rightarrow 0 \quad(\text { as } \delta \rightarrow 0), \\
& \left|\varepsilon^{\alpha / 2} v_{\delta}-\varepsilon^{\alpha / 2} v\right|_{C^{\alpha, \alpha / 2}\left(\bar{\Omega}_{T}\right)} \rightarrow 0 \quad(\text { as } \delta \rightarrow 0) .
\end{aligned}
$$

Suppose that $\bar{\rho}_{\delta \lambda}$ is the unique solution of the following periodic Cauchy problem of the parabolic equation

$$
\begin{gathered}
\partial_{t} \bar{\rho}_{\delta \lambda}=v_{\delta} \sqrt{1+\sum_{i=1}^{2}\left|\varphi_{\delta}^{(i)}\right|^{2}\left(\partial_{\omega_{i}} \bar{\rho}_{\delta \lambda}\right)^{2}}+\lambda \Delta_{\omega} \bar{\rho}_{\delta \lambda} \quad \text { in } \Gamma_{0 T} \\
\bar{\rho}_{\delta \lambda}=0 \quad \text { on } t=0
\end{gathered}
$$

where $\lambda>0$ is a small parameter, and $\Delta_{\omega}$ is the Laplace operator in $\mathbb{R}_{\omega}^{2}$.

Construct comparison functions $\pm M_{0} t$; here $M_{0}$ is from the estimate (3.9). From the comparison principle (see [11], p. 52) we have

$$
\left|\bar{\rho}_{\delta \lambda}\right|_{L^{\infty}\left(\Gamma_{0 T}\right)} \leq M_{0} T
$$

consequently, we choose $T$ to be small enough such that

$$
\left|\bar{\rho}_{\delta \lambda}\right|_{L^{\infty}\left(\Gamma_{0 T}\right)} \leq \gamma_{0} / 4
$$

To obtain further estimates, we differentiate Eq. (3.14) with respect to $w_{j}, j=1,2$, and set $p_{j}=\partial_{\omega_{j}} \bar{\rho}_{j \lambda}, p_{i j}=\partial_{\omega_{i}} \partial_{\omega}, \bar{\rho}_{\delta \lambda}, i, j=1,2$. Then

$$
\begin{aligned}
\partial_{t} p_{j}=( & \left.\partial_{\omega_{j}} v_{\delta}\right) \sqrt{1+\sum_{i=1}^{2}\left|\varphi_{\delta}^{(t)}\right|^{2} p_{t}^{2}} \\
& +v_{\delta} \sum_{i=1}^{2}\left(\varphi_{\delta}^{(i)} \cdot \partial_{\omega_{i}} \varphi_{\delta}^{(i)} p_{i}^{2}+\left|\varphi_{\delta}^{(i)}\right|^{2} p_{i} p_{i j}\right) /\left(1+\sum_{i=1}^{2}\left|\varphi_{\delta}^{(i)}\right|^{2} p_{i}^{2}\right)^{1 / 2} \\
& +\lambda \Delta_{\omega} p_{j}, \quad j=1,2 .
\end{aligned}
$$

Multiply the above equation by $2 p_{j}$ and sum with respect to $j$. Notice that

$$
2 \sum_{j=1}^{2} p_{j} p_{i j}=\partial_{\omega_{i}}\left(p_{1}^{2}+p_{2}^{2}\right) \text {. }
$$

Set $P=p_{1}^{2}+p_{2}^{2}$ and notice that

$$
\begin{gathered}
2 p_{j}\left(\partial_{\omega_{j}} v_{\delta}\right) \sqrt{1+\sum_{i=1}^{2}\left|\varphi_{\delta}^{(i)}\right|^{2} p_{i}^{2}} \leq C(P+1), \quad j=1,2 \\
2 p_{j} v_{\delta}\left(\sum_{i=1}^{2} \varphi_{\delta}^{(i)} \partial_{\omega_{i}} \varphi_{\delta}^{(i)} p_{i}^{2}\right) /\left(1+\sum_{i=1}^{2}\left|\varphi_{\delta}^{(i)}\right|^{2} p_{i}^{2}\right)^{1 / 2} \leq C P, \quad j=1,2
\end{gathered}
$$


and

$$
\sum_{i=1}^{2} 2 p_{j} \Delta_{\omega} p_{j}=\Delta_{\omega} P-\sum_{i, j=1}^{2} p_{i j}^{2} \leq \Delta_{\omega} P .
$$

The above calculations reduce to

$$
\left\{\begin{array}{l}
\partial_{t} P \leq \lambda \Delta_{\omega} P+v_{\delta} \sum_{i=1}^{2} \frac{\left|\varphi_{\delta}^{(i)}\right|^{2} p_{i}}{\left(1+\sum_{i=1}^{2}\left|\varphi_{\delta}^{(i)}\right|^{2} p_{i}^{2}\right)^{1 / 2}} \partial_{\omega_{i}} P+C_{1} P+C_{2}, \\
P(\omega, 0)=0
\end{array}\right.
$$

where $C_{i}, i=1,2$, are constants that are independent of $\lambda$ and $p_{i}, p_{i j}, P$. Notice that $v_{\delta} \frac{\left|\varphi_{\delta}^{(i)}\right|^{2} p_{i}}{\left(1+\sum_{i=1}^{2}\left|\varphi_{\delta}^{(i)}\right|^{2} p_{i}^{2}\right)^{1 / 2}}, i=1,2$, are also bounded functions. So if we choose $C=C\left(M_{2+\alpha}\right.$, $N)$ big enough, and let $C\left(M_{2+\alpha}, N\right) t$ be the comparison function, then

$$
P \leq C\left(M_{2+\alpha}, N\right) T .
$$

Similarly, by differentiating (3.14) twice with respect to $\omega_{i}$ and $\omega_{j}, i, j=1,2$, and using the estimate (3.19) we can get

$$
\left|D_{\omega}^{2} \bar{\rho}_{\delta \lambda}\right| \leq C\left(M_{2+\alpha}, N\right) T .
$$

Since $v_{\delta}$ and $\varphi_{\delta}^{(i)}, i=1,2$, are $C^{\infty}$ in the variables $\omega_{1}$ and $\omega_{2}$, we can use successive differentiations in their variables and the comparison function as before to get $L^{\infty}$ estimates on $D_{\omega}^{2} \bar{\rho}_{\delta \lambda}$ depending on $\delta$ but not on $\lambda$. Hence we can let $\lambda \rightarrow 0$ in (3.14) to get that there exists $\bar{\rho}_{j}$ such that $\partial_{\omega_{i}} \bar{\rho}_{\delta \lambda} \rightarrow \partial_{\omega_{i}} \bar{\rho}_{\delta} \equiv q_{i}, \partial_{\omega_{i}} \partial_{\omega_{j}} \bar{\rho}_{\delta \lambda} \rightarrow \partial_{\omega_{i}} \partial_{\omega_{j}} \bar{\rho}_{\delta} \equiv q_{i j}$ and

$$
\left\{\begin{array}{l}
\partial_{t} q_{i j}-\sum_{k=1}^{2} \frac{v_{\delta}\left|\varphi_{\delta}^{(k)}\right|^{2} q_{k}}{\sqrt{1+\sum_{i=1}^{2}\left|\varphi_{\delta}^{(i)}\right|^{2} q_{l}^{2}}} \partial_{\omega_{k}} q_{i j}=F_{i j} \quad \text { in } \Gamma_{0 T} \\
\left.q_{i j}\right|_{t=0}=0 \quad(i, j=1,2)
\end{array}\right.
$$

where the functions $F_{i j}=F_{i j}(\omega, t)$ are functions given in terms of $v_{\delta}, \nabla_{\omega} v_{\delta}, D_{\omega}^{2} v_{\delta}, \varphi_{\delta}^{(i)}$, $\nabla_{\omega} \varphi_{\delta}^{(i)}, D_{\omega}^{2} \varphi_{\delta}^{(i)}, q_{i}, q_{i j}, i, j=1,2$.

In order to get the Hölder regularity of $q_{i j}$ with respect to $\omega$, we introduce the characteristics of (3.21):

$$
\left\{\begin{array}{l}
\frac{d \xi_{i}}{d t}=-\frac{v_{\delta}\left|\varphi_{\delta}^{(i)}\right|^{2} q_{l}}{\sqrt{1+\sum_{l=1}^{2}\left|\varphi_{\delta}^{(l)}\right|^{2} q_{l}^{2}}}(\omega, t) \\
\xi_{i}(0)=\omega_{i}, \quad i=1,2
\end{array}\right.
$$

Suppose the solution of the above ordinary differential system is

$$
\xi_{i}=\xi_{i}(\omega, t), \quad i=1,2 .
$$

Therefore,

$$
\left(\frac{\partial \xi_{i}}{\partial \omega_{j}}\right)_{2 \times 2}=\exp \left\{\int_{0}^{t}\left(\frac{\partial \beta_{i}}{\partial \omega_{j}}\right)_{2 \times 2}(\omega, \tau) d \tau\right\}
$$

where $\beta_{i}(\omega, t)=-\frac{v_{\delta}\left|\varphi_{\delta}^{(i)}\right|^{2} q_{i}}{\sqrt{1+\sum_{l=1}^{2}\left|\varphi_{\delta}^{(l)}\right|^{2} q_{i}^{2}}}(\omega, t), i=1,2$. So if we let $T$ be small enough, then

$$
\frac{1}{2} \leq\left|\left(\frac{\partial \xi_{i}}{\partial \omega_{j}}\right)_{2 \times 2}\right| \leq 2
$$


Rewriting the system (3.21) in integral form along the characteristics, we have, for any $\omega, \bar{\omega} \in \mathbb{R}^{2}, 0<t<T$,

$$
\begin{aligned}
& \left|q_{i j}(\xi(\omega, t), t)-q_{i j}(\xi(\bar{\omega}, t), t)\right| \\
& \quad \leq C \int_{0}^{t}\left[\sum_{i, j=1}^{2}\left|q_{i j}(\xi(\omega, \tau), \tau)-q_{i j}(\xi(\bar{\omega}, \tau), \tau)\right|+|\xi(\omega, \tau)-\xi(\bar{\omega}, \tau)|\right] d \tau
\end{aligned}
$$

where $C$ is independent of $\delta$.

Summing up the above inequality with respect to $i$ and $j$ using the Gronwall inequality, we can obtain that there is a constant $C$ that is independent of $\delta$, but depends on $M_{2+\alpha}$ and $N$, such that

$$
\left|q_{i j}\right|_{C\left([0, T] ; C^{\alpha}\left(\Gamma_{0}\right)\right)} \leq C\left(M_{2+\alpha}, N\right) T, \quad i, j=1,2 .
$$

By the equation

$$
\partial_{t} \bar{\rho}_{\delta}=v_{\delta} \sqrt{1+\sum_{i=1}^{2}\left|\varphi_{\delta}^{(i)}\right|^{2}\left(\partial_{\omega_{i}} \bar{\rho}_{\delta}\right)^{2}}
$$

we know that

$$
\begin{aligned}
&\left|\partial_{t} \bar{\rho}_{\delta}\right|_{L^{\infty}\left(\Gamma_{0 T}\right)} \leq M_{0}\left[1+C\left(M_{2+\alpha}, N\right) T\right] \\
&\left|\partial_{t} \bar{\rho}_{\delta}\right|_{B\left([0, T] ; C^{\alpha}\left(\Gamma_{0}\right)\right)} \leq C\left(M_{\alpha}\right)\left[1+C\left(M_{2+\alpha}, N\right) T\right] .
\end{aligned}
$$

If $T$ is small enough such that

$$
C\left(M_{2+\alpha}, N\right) T \leq 1
$$

we have the following estimates:

$$
\begin{aligned}
\left|\partial_{t} \bar{\rho}_{\delta}\right|_{L^{\infty}\left(\Gamma_{0 T}\right)} & \leq 2 M_{0}, \\
\left|\partial_{t} \bar{\rho}_{\delta}\right|_{B\left([0, T] ; C^{\alpha}\left(\Gamma_{0}\right)\right)} & \leq 2 C\left(M_{\alpha}\right),
\end{aligned}
$$

where $N$ is to be determined later on, which will be independent of $T$.

The estimates (3.16), (3.19), (3.20), and (3.23) reduce to

$$
\left|\bar{\rho}_{\delta}\right|_{B\left([0, T] ; C^{2+\alpha}\left(\Gamma_{0}\right)\right)} \leq M_{0}
$$

if $T$ is small enough.

Applying the following interpolation inequality, where the embedding constant $C_{\alpha}$ is independent of $T$ (see [13], Lemma 5.1.1),

$$
\begin{aligned}
& \left|\nabla_{\omega} \bar{\rho}_{\delta}\right|_{C^{0,1+\alpha / 2}\left(\Gamma_{0 T}\right)}+\left|D_{\omega}^{2} \bar{\rho}_{\delta}\right|_{C^{0, \alpha / 2}\left(\Gamma_{0 T}\right)} \\
& \quad \leq C_{\alpha}\left(\left|\bar{\rho}_{\delta}\right|_{B\left([0, T] ; C^{2+\alpha}\left(\Gamma_{0}\right)\right)}+\left|\partial_{t} \bar{\rho}\right|_{B\left([0, T] ; C^{\alpha}\left(\Gamma_{0}\right)\right)}\right) \\
& \quad \leq C_{\alpha}\left[M_{0}+2 C\left(M_{\alpha}\right)\right] .
\end{aligned}
$$

Combining (3.28) and (3.29) we obtain

$$
\begin{gathered}
\left|\nabla_{\omega} \bar{\rho}_{\delta}\right|_{C^{1+\alpha,(1+\alpha) / 2}\left(\Gamma_{0 T}\right)}+\left|D_{\omega}^{2} \bar{\rho}_{\delta}\right|_{C^{\alpha, \alpha / 2}\left(\Gamma_{0 T}\right)} \\
\leq M_{0}+C_{\alpha}\left[M_{0}+2 C\left(M_{\alpha}\right)\right] \equiv N_{1} .
\end{gathered}
$$


Multiplying (3.24) by $\varepsilon^{\alpha / 2}$ to get

$$
\varepsilon^{\alpha / 2} \partial_{t} \bar{\rho}_{\delta}=\left(\varepsilon^{\alpha / 2} v_{\delta}\right) \sqrt{1+\sum_{i=1}^{2}\left|\varphi_{\delta}^{(i)}\right|^{2}\left(\partial_{\omega_{i}} \bar{\rho}_{\delta}\right)^{2}}
$$

and recalling the estimates (3.10) and (3.30) we have

$$
\left|\varepsilon^{\alpha / 2} \partial_{t} \bar{\rho}_{\delta}\right|_{C^{\alpha, \alpha / 2}\left(\Gamma_{0 T}\right)} \leq C\left(M_{\alpha}\right)\left\{1+M_{0}+C_{\alpha}\left[M_{0}+2 C\left(M_{\alpha}\right)\right]\right\} \equiv N_{2} .
$$

So we can choose $N=\max \left\{N_{1}, N_{2}\right\}$, and then let $T$ be small enough such that (3.25) is true.

Passing to the limit as $\delta \rightarrow 0$, through an appropriate subsequence, we obtain a function $\bar{\rho} \in D$, which turns out to be the (unique) solution to (3.12), (3.13), and still satisfies the estimates (3.17), (3.26), (3.28), and (3.30), (3.31).

Define a mapping $\mathcal{F}: D \rightarrow D$ by

$$
\mathcal{F}(\rho)=\bar{\rho} .
$$

If we consider the uniform topology in $D$, then $D$ is a closed, convex, and compact subset of the Banach space $C^{2+\alpha, 1+\alpha / 2}\left(\bar{\Omega}_{T}\right)$. The continuity of $\mathcal{F}$ follows by compactivity of $D$ and the uniqueness of the mapping $\mathcal{F}$. Therefore the Shauder fixed point theorem yields the existence of

$$
\rho=\mathcal{F}(\rho) \in D,
$$

which solves the problem $(2.1)-(2.5)$.

This completes the proof of Lemma 3.1 as well as Theorem 2.1.

4. The convergence of the solutions. In the previous section we have proved that the problem (2.1)-(2.5) has a unique solution $\rho=\rho_{\varepsilon}(\omega, t) \in D$ for fixed $\varepsilon>0$. Let $v=v_{\varepsilon}$. Then $\left(v_{\varepsilon}, \rho_{\varepsilon}\right)$ satisfy

$$
\begin{array}{ll}
\varepsilon \partial_{t} v_{\varepsilon} & -\sum_{i, j=1}^{3} a_{i j}\left(\rho_{\varepsilon}, \nabla_{\omega} \rho_{\varepsilon}\right) \frac{\partial^{2} v_{\varepsilon}}{\partial y_{i} \partial y_{j}} \\
\quad+\sum_{i, j=1}^{3} a_{i}\left(\rho_{\varepsilon}, \nabla_{\omega} \rho_{\varepsilon}, D_{\omega}^{2} \rho_{\varepsilon}, \varepsilon \partial_{t} \rho_{\varepsilon}\right) \frac{\partial v_{\varepsilon}}{\partial y_{i}}=0 & \text { in } \Omega_{T}, \\
v_{\varepsilon}=g(y, t) & \text { on } \Gamma_{1 T}, \\
v_{\varepsilon}(y, 0)=u_{0}(y) & \text { on } t=0, \\
S_{\rho_{t}} \partial_{\eta} v_{\varepsilon}-\sum_{i=1}^{2} \partial_{\omega_{i}} \rho_{\varepsilon}\left|\varphi^{(i)}\left(\omega, \rho_{\varepsilon}\right)\right|^{2} \partial_{\omega_{i}} v_{\varepsilon}+S_{\rho_{\varepsilon}}^{1 / 2} v_{\varepsilon}=0 & \text { on } \Gamma_{0 T}, \\
\partial_{t} \rho_{\varepsilon}=S_{\rho_{\varepsilon}}^{1 / 2} v_{\varepsilon} & \text { on } \Gamma_{0 T},
\end{array}
$$

and uniform estimates

$$
\begin{aligned}
&\left|\rho_{\varepsilon}\right|_{B\left([0, T] ; C^{2+\alpha}\left(\Gamma_{0}\right)\right)} \leq C, \\
&\left|\partial_{t} \rho_{\varepsilon}\right|_{B\left([0, T] ; C^{\alpha}\left(\Gamma_{0}\right)\right)} \leq C, \\
&\left|v_{\varepsilon}\right|_{B\left([0, T] ; C^{2+\alpha}(\bar{\Omega})\right)} \leq C .
\end{aligned}
$$


TheOREM 4.1. Under the assumptions (2.6)-(2.11),

(1) (The convergence of free boundaries) there is a function

$$
\rho(\omega, t) \in B\left([0, T] ; C^{2+\alpha}\left(\Gamma_{0}\right)\right), \quad \partial_{t} \rho \in B\left([0, T] ; C^{\alpha}\left(\Gamma_{0}\right)\right)
$$

such that

$$
\rho_{\varepsilon} \rightarrow \rho \quad \text { in } C\left([0, T] ; C^{2+\beta}\left(\Gamma_{0}\right)\right), 0<\beta<\alpha .
$$

(2) (The convergence of solutions) If $\Delta u_{0}(x)=0$ in $\Omega$, then

$$
\left|\partial_{t} v_{\varepsilon}\right|_{L^{\infty}\left(\Omega_{T}\right)} \leq C
$$

Moreover, there is a function $v(x, t) \in B\left([0, T] ; C^{2+\alpha}(\bar{\Omega})\right)$ such that

$$
v_{\varepsilon} \rightarrow v \quad \text { in } C\left([0, T] ; C^{2+\beta}(\bar{\Omega})\right) .
$$

(3) $(v, \rho)$ is the classical solution for the Hele-Shaw problem with a kinetic condition at the free boundary.

Proof. (1) (4.9) follows by the estimates (4.6) and (4.7) and Simon's compact theorem (see [12], Corollary 4, p. 85).

(2) In order to get $(4.10)$, let us go back to the $(x, t)$ coordinates

$$
\begin{array}{ll}
\varepsilon \partial_{t} u_{\varepsilon}-\Delta u_{\varepsilon}=0 & \text { in } \Omega_{\rho T}, \\
u_{\varepsilon}=g(x, t) & \text { on } \Gamma_{1 T}, \\
u_{\varepsilon}(x, 0)=u_{0}(x) & \text { on } t=0 \\
\frac{\partial u_{\varepsilon}}{\partial n}+u_{\varepsilon}=0 & \text { on } \Gamma_{\rho T} \\
V_{n}=u_{\varepsilon} & \text { on } \Gamma_{\rho T} .
\end{array}
$$

Here $u_{\varepsilon}(x, t)=v_{\varepsilon}\left(\ell_{\rho T}^{-1}(x, t)\right)$ and by $(4.8)$,

$$
\left|u_{\varepsilon}\right|_{C\left([0, T] ; C^{2+\alpha}(\bar{\Omega}(t))\right)} \leq C .
$$

Differentiating with respect to $t$ in (4.12)-(4.15), we have

$$
\begin{array}{ll}
\varepsilon \partial_{t}\left(\partial_{t} u_{\varepsilon}\right)-\Delta\left(\partial_{t} u_{\varepsilon}\right)=0 & \text { in } \Omega_{\rho T}, \\
\partial_{t} u_{\varepsilon}=\partial_{t} g(x, t) & \text { on } \Gamma_{1 T}, \\
\partial_{t} u_{\varepsilon}=0 & \text { on } t=0, \\
\frac{\partial\left(\partial_{t} u_{\varepsilon}\right)}{\partial n}+\partial_{t} u_{\varepsilon}+V_{n}\left|\nabla\left(\frac{\partial u_{\varepsilon}}{\partial n}+u_{\varepsilon}\right)\right|=0 . &
\end{array}
$$

Substituting (4.16) into the last equality to get

$$
\frac{\partial\left(\partial_{t} u_{\varepsilon}\right)}{\partial n}+\partial_{t} u_{\varepsilon}=-u_{\varepsilon}\left|\nabla\left(\frac{\partial u_{\varepsilon}}{\partial n}+u_{\varepsilon}\right)\right| \quad \text { on } \Gamma_{\rho T},
$$

and applying the maximum principle to the system (4.18)-(4.20) and (4.22), using (4.17) we get

$$
\left|\partial_{t} u_{\varepsilon}\right|_{L^{\infty}\left(\Omega_{\rho T}\right)} \leq C\left(\left|\partial_{t} g\right|_{L^{\infty}\left(\Gamma_{1 T}\right)}+\left|u_{\varepsilon}\right|_{C\left([0, T] ; C^{2}(\Omega(t))\right)}\right) \leq C ;
$$

here $C$ is independent of $\varepsilon>0$. 
Considering

we get

$$
\partial_{t} u_{\varepsilon}=\partial_{t} v_{\varepsilon}+\nabla_{y} v_{\varepsilon} \cdot \frac{\partial \ell_{\rho T}^{-1}}{\partial t}
$$

$$
\left|\partial_{t} v_{\varepsilon}\right|_{L^{\infty}\left(\Omega_{T}\right)} \leq\left|\partial_{t} u_{\varepsilon}\right|_{L^{\infty}}+C\left|\nabla_{y} v\right|_{L^{\infty}}\left|\partial_{t} \rho_{\varepsilon}\right|_{L^{\infty}} \leq C
$$

where $C$ is independent of $\varepsilon>0$. From the estimates (4.8) and (4.23) we get (4.11) by the use of Simon's compact theorem again.

(3) From (4.5) we know that

$$
\partial_{t} \rho_{\varepsilon} \rightarrow \partial_{t} \rho \quad \text { in } C\left([0, T] ; C^{1+\beta}\left(\Gamma_{0}\right)\right) .
$$

Letting $\varepsilon \rightarrow 0$ in (4.1), (4.2), (4.4), and (4.5) we get

$$
\begin{array}{ll}
-\sum_{i, j=1}^{3} a_{i j}\left(\rho, \nabla_{\omega} \rho\right) \frac{\partial^{2} v}{\partial y_{i} \partial y_{j}}+\sum_{i, j=1}^{3} a_{i}\left(\rho, \nabla_{\omega} \rho, D_{\omega}^{2} \rho\right) \frac{\partial v}{\partial y_{i}}=0 & \text { in } \Omega_{T} \\
v=g(x, t) & \text { on } \Gamma_{1 T}, \\
S_{\rho} \partial_{\eta} v-\sum_{i=1}^{2} \partial_{\omega_{i}}\left|\varphi^{(i)}(\omega, \rho)\right|^{2} \partial_{\omega_{i}} v+S_{\rho}^{1 / 2} v=0 & \text { on } \Gamma_{0 T} \\
\partial_{t} \rho=S_{\rho_{\varepsilon}}^{1 / 2} v & \text { on } \Gamma_{0 T} .
\end{array}
$$

This system means that $(v, \rho)$ is the classical solution of the Hele-Shaw problem with a kinetic condition at the free boundary.

This completes the proof of Theorem 4.1.

REMARK. The $\Delta u_{0}(x)=0$ in $\Omega$ is the necessary condition for the convergence of solutions because the solution of the Hele-Shaw problem must satisfy this condition.

\section{REFERENCES}

[1] A. Visintin, Stefan problem with a kinetic condition at the free boundary, Ann. Mat. Pura Appl. 146, 97-122 (1987)

[2] W. Xie, The Stefan problem with a kinetic condition at the free boundary, SIAM J. Math. Anal. 21, 362-373 (1990)

[3] A. Friedman and B. Hu, Stefan problem for multi-dimensional reaction diffusion systems, SIAM J. Math. Anal. 27, 1212-1234 (1996)

[4] E. V. Radkevich and B. O. Ėtonkulov, On the existence of a classical solution to the problem of the swelling of glassy polymers, Math. Notes 57, 615-624 (1995)

[5] E. Hanzawa, Classical solution of the Stefan problem, Tohoku Math. J. 33, 297-335 (1981)

[6] A. Friedman and B. Hu, The Stefan problem with kinetic condition at the free boundary, Ann. Scuola Norm. Pisa 19, 87-111 (1992)

[7] Yu. E. Hohlov and M. Reissig, On classical solvability for the Hele-Shaw moving boundary problems with kinetic undercooling regularization, European J. Appl. Math. 6, 421-439 (1995)

[8] J. F. Rodrigues (ed.), Free Boundary Problems News 4, 11-12, University of Lisboa, 1994

[9] F. Yi. Asymptotic behaviour of the solutions of the supercooled Stefan problem, Proceedings of the Royal Society of Edinburgh 127 A, 181-190 (1997)

[10] E. V. Radkevich, On conditions for the existence of a classical solution of the modified Stefan problem, Russian Acad. Sci. Sb. Math. 75, 221-246 (1993)

[11] A. Friedman, Partial Differential Equations of Parabolic Type, Prentice-Hall, Englewood Cliffs, NJ, 1964

[12] J. Simon, Compact sets in the space $L^{p}(0, T ; B)$, Ann. Mat. Appl. 146, 65-96 (1987)

[13] A. Lunardi, Analytic Semigroups and Optimal Regularity in Parabolic Problems, Birkhäuser, 1995 